chronic pancreatic changes 5/17 (29.4\%), $p=0.004$. Analyses of other markers did not show significant differences in proportions of biochemical marker deficiencies between PEI and non-PEI groups. Using selenium $(<0.61 \mu \mathrm{mol} / \mathrm{l})$ as a marker of PEI in high risk groups gave a sensitivity, specificity, positive predictive value and negative predictive value of $25.81 \%$ (95\% CI=12.3\%-38.5\%)， 92.45\% (95\% CI=83\%-98\%), $66.66 \% \quad(95 \% \quad \mathrm{CI}=39.3 \%-86 \%), \quad 68.06 \% \quad(95 \% \quad \mathrm{CI}=58.9 \%-$ $67 \%)$, respectively.

Conclusion Although a selenium deficiency was significantly associated with PEI, its poor sensitivity as a test for PEI would only make it supportive of a diagnosis and other tests would be required. Apart from selenium levels it would appear that checking serum nutritional markers for suspected cases of PEI at diagnosis results in a low diagnostic yield. PEI patients are overweight suggesting that using a low BMI to target patients is insufficient to prompt testing for PEI or malnutrition in this patient group.

\section{PWE-068 PREVALENCE AND OUTCOMES OF PANCREATIC CYSTIC LESIONS IN LIVER TRANSPLANT RECIPIENTS: A PILOT STUDY FROM A LIVER TRANSPLANT CENTRE}

Margaret (Geri) Keane*, David Reffitt, Philip Harrison, John Devlin, Deepak Joshi. Kings College Hospital, London, UK

\subsection{6/gutjnl-2019-BSGAbstracts.299}

Introduction Incidental small pancreatic cystic lesions (PCLs) are common present in $1-2 \%$ of the population undergoing CT for non-pancreatic indications. They are increasingly being identified on preoperative imaging of patients undergoing orthotopic liver transplantation (OLT). Most PCL are benign but a proportion, namely intraductal papillary mucinous neoplasms (IPMNs) and mucinous cystic neoplasms (MCNs) harbour malignant potential. There is growing evidence that liver transplant recipients are at increased risk of extrahepatic malignancy. Whether the natural history of PCL in liver transplant recipients is the same as the general population in uncertain. The aim of this study was to look at the incidence and progression of PCL in liver transplant recipients.

Methods A pilot series of patients from a large UK liver transplant centre, were included. Between January 1990 and December 2017, a proportion of cases were selected from our consecutive database of liver transplant patients.

Results 238 patients were included. PCL were present in $4 \%$ (9/238), 8/9 were male. End stage liver disease was due to alcohol related liver disease in 5 cases, Primary Sclerosing Cholangitis in 3 and was unknown in 1. Follow-up was undertaken by MRI annually. During the median follow up of 79 months (range 33-288 months) all PCL in the liver transplant patients on long-term immunosuppression remained stable in size. One developed mild dilation of the main pancreatic duct but no other features of concern were observed.

Conclusion The prevalence of PCLs in liver transplant recipients was similar to that of the general population. PCL do not appear to have an accelerated malignant potential in post liver transplant patients, indicating the current International and European surveillance guidelines are likely to be applicable in this group.

\section{PWE-069 MANAGEMENT OF PANCREATIC FLUID COLLECTIONS BY LAMS: LARGE SERIES FROM A TERTIARY REFERRAL HPB CENTRE}

Margaret (Geri) Keane*, Yasser El-Sherif, Ben Warner, David Reffitt, Phillip Harrison, Deepak Joshi, John Devlin. Kings College Hospital, London, UK

\subsection{6/gutjnl-2019-BSGAbstracts.300}

Background Endoscopic transmural drainage is increasingly accepted as the first-line treatment for patients with symptomatic pancreatic fluid collections (PFC). International data has shown that in comparison to double pigtail stents Lumen Apposing Metal Stents (LAMS) are associated with higher rates of clinical success (especially in cysts with necrosis), shorter procedure times and potentially fewer adverse events. To date there has been limited data from the UK on the utility of these stents.

Methods Between January 2016 - December 2018 all patients who required endoscopic drainage of a pancreatic fluid collection via EUS-guided LAMS (Hot AXIOS ${ }^{\mathrm{TM}}$ system, Boston Scientific) were included. Treatment success, length of hospital stay, adverse events, reinterventions and length of follow-up were recorded in each case.

Results During the 3 year study period, 84 EUS-guided LAMS were placed on 80 patients. Median age was 52 years (Range 7-79 years). 52\% (44/84) were female. Necrotic material was present in $44 \%(37 / 84)$ and significant portal hypertension visible on EUS in $8 \%(7 / 84)$. In four patients a $8 \mathrm{~mm}$ stent was placed, $10 \mathrm{~mm}$ stents were placed in thirty-five patients, a $15 \mathrm{~mm}$ stent in twenty-five patients and a $20 \mathrm{~mm}$ stent in eleven patients (in nine patients the stent size was unknown). Adverse events occurred in 11\% (9/84) of cases (6 cases of failed stent deployment, 2 buried stents and one recurrent pancreatic fluid collection following stent removal)

Conclusion Use of LAMSs in the management of pancreatic fluid collections was safe and effective and associated with low rates of cyst recurrence.

\section{PWE-070 TRENDS IN PANCREATIC CYSTIC LESIONS REFERRED FOR ENDOSCOPIC ULTRASOUND: 10 YEARS EXPERIENCE}

${ }^{1}$ John Leeds*, ${ }^{1}$ Manu Nayar, ${ }^{2}$ Sarah Johnson, ${ }^{2}$ Beate Haugk, ${ }^{2}$ Antony Darne, ${ }^{1}$ Kofi Oppong. ${ }^{1}$ Freeman Hospital, Newcastle Upon Tyne, UK; ${ }^{2}$ Royal Victoria Informary, Newcastle Upon Tyne, UK

\subsection{6/gutjnl-2019-BSGAbstracts.301}

Introduction Pancreatic cystic lesions are being identified more frequently as cross sectional imaging has improved. Referral for endoscopic ultrasound (EUS) for further assessment and possible sampling is well recognised however there is little data on whether the characteristics of such lesions have changed over time. There is also a paucity of data concerning changes in pathological findings and surgical resection rates with respect to cystic lesions. The aim of this study was to assess trends in referral for EUS with respect to patient and cyst characteristics, pathological adequacy and surgical resection rates over a 10 year period to our centre.

Patients and methods Retrospective analysis of the EUS database was performed over the period 2003 to 2012. EUS procedures for assessment of cystic lesions were identified and 